Saudi Journal of Oral and Dental Research

Abbreviated Key Title: Saudi J Oral Dent Res

ISSN 2518-1300 (Print) |ISSN 2518-1297 (Online)

Scholars Middle East Publishers, Dubai, United Arab Emirates

Journal homepage: https://saudijournals.com

\title{
Effectiveness of MI Varnish in Preventing White Spot Lesions during Orthodontic Treatment -In Vivo Study
}

Dr. Irfan Habeeb, MDS ${ }^{1}$, Dr. K. Nillan Shetty, MDS$^{2}$, Dr. Harshitha V, MDS ${ }^{3}$, Dr. Shetty Suhani Sudhakar, MDS ${ }^{4 *}$, Dr. Hannah Sarah, BDS ${ }^{5}$

${ }^{1}$ Private Practitioner, Kasargod, Kerala, India

${ }^{2}$ Principal and Head of department of Orthodontics, A.J. Institute of Dental sciences, Mangalore, Karnataka, India

${ }^{3}$ Department of Orthodontics, A.J. Institute of Dental sciences, Mangalore, Karnataka, India

${ }^{4}$ Private practitioner, Bangalore, Karnataka, India

${ }^{5}$ Department of prosthodontics, Yenepoya dental college, Mangalore, Karnataka

DOI: $10.36348 /$ sjodr.2022.v07i01.003 $\quad$ | Received: 04.12.2021 | Accepted: 06.01.2022 | Published: 12.01 .2022

*Corresponding author: Dr. Shetty Suhani Sudhakar

Private practitioner, Bangalore, Karnataka, India

\section{Abstract}

Objective: The objective was to evaluate the efficacy of MI varnish in preventing white spot lesions in patients with fixed orthodontic therapy. Method The study was performed where 100 patients undergoing orthodontics treatment were allocated randomly to 2 subgroups with differing frequencies of MI varnish application. Degree of mineralisation was measured on the vestibular surfaces of 12 teeth ( 6 varnish \& 6 unvarnished controls) Measurement were taken at 4 sites using camera EOS 750D and then subjected to statistical analysis. Results: The statistical analysis showed difference in the degree of demineralization between treated and untreated teeth, and was statistically significant in terms of time point, frequency of application or specific tooth site. Conclusion: Periodic application of MI varnish can offer some protection against white spot lesions and it is not statistically significant degree if patients have excellent oral hygiene. Additional protection is conferred by applying the product every 3 months compared with every 6 months

Keywords: White spot lesion, MI Varnish.

Copyright (C) 2022 The Author(s): This is an open-access article distributed under the terms of the Creative Commons Attribution 4.0 International License (CC BY-NC 4.0) which permits unrestricted use, distribution, and reproduction in any medium for non-commercial use provided the original author and source are credited.

\section{INTRODUCTION}

White spot lesions have been defined as "subsurface enamel porosity from carious demineralization" that presents as a milky white opacity when located on smooth surfaces [1]. The initial carious lesions are the so -called "white spot" lesions, which imply that there is a subsurface area with most of the mineral loss, beneath a relatively intact enamel surface.

Clinically, early caries lesion in enamel is initially seen as a white opaque spot and is characterized by being softer than the adjacent sound enamel wand is increasingly whiter when dried with air.

In general, orthodontic patients have significantly more WSLs than non-orthodontic patients, and these WSLs may present esthetic problems years after treatment. The caries process takes place slowly which requires repeated episodes of prolonged exposure to acidic conditions consistently below the critical $\mathrm{pH}$ for enamel dissolution ( $\mathrm{pH} 5.5$, demineralization) with intervening periods of return to the resting $\mathrm{pH}$ of plaque( $\mathrm{pH} 7.0$, remineralisation period) [1].

Aim and objectives of the study was to evaluate the efficacy of a MI varnish in preventing white spot lesions and to assess any correlation between the degree of demineralization and the length of the observation period, to determine frequency of incidence with respect to each quadrant, to determine frequency of incidence with respect to frequency of varnish application and to assess any correlation between degree of demineralization with respect to the tooth site.

\section{MATERIALS AND METHODS}

The study was done at the Department of Orthodontics and Dentofacial Orthopaedics. The study included 100 subjects. A split-mouth study design was 
used for 100 orthodontic patients, allocated randomly to 2 subgroups with various frequencies of GC MI Varnish application.

Repeated measurements of the degree of demineralization was taken on the vestibular surfaces of 12 teeth (6 varnished and 6 unvarnished controls). Measurements was taken at 4 areas using Digital Vernier caliper and then subjected to statistical analysis.

For all the 100 patients, consent was taken regarding the MI Varnish application. This prospective study was approved by the Research Ethics committee of the Institutional Ethics Committee.

Inclusion criteria were iindividuals' with fixed orthodontic appliances, complete permanent dentition till erupted second molar, no caries or fillings in maxillary and mandibular arch, no hypo calcified teeth, no partially erupted teeth. Exclusion criteria were dental fluorosis in maxillary and mandibular teeth, endocrine disorders, endodontically treated teeth and patients with syndromes.

Hundred Individuals all scheduled for fixed orthodontic treatment at AJ Institute of Dental Sciences, Department of Orthodontics will be selected in this study. The sample consisted of 100 individuals including males and females .A splitmouth study will be conducted, applying the varnish to quadrants 1 and 3 , with quadrants 2 and 4 as the untreated controls.

Opposing quadrants will be chosen to eliminate any bias, which can affect the uniformity of brushing accuracy. The 6 varnished and the 6 control teeth will be the central incisors, the canines, and the second premolars: i.e., teeth from each of the maxillary and mandibular anterior, medial, and posterior sectors. The patients will be assigned to 2 sub-groups according to the frequency of MI Varnish application:

Group 1, comprising 50 patients (including males and females), in whom MI Varnish will be applied for every 3 months; and Group2, comprising 50 patients (including males and females), in whom MI Varnish will be applied every 6 months.
Both groups will be given a professional scaling and polishing. All patients will receive a hygiene kit that will contain a medium-bristle tooth brush. Before the trial all patients will have a dental examination with caries and periodontal evaluation. An oral hygiene session with motivation by the dental hygienist will be conducted.

Patient's: Occlusal/ incisal, Gingival, Mesial Distal to the brackets as Initial values (T0) will be recorded. After the initial readings the dental arches will be isolated, and the surfaces will be allowed to dry.

MI Varnish will be applied to the enamel around the brackets of the maxillary right central incisor, canine, and second premolar and the mandibular left central incisor, canine, and second premolar using a microbrush.

After about 5 minutes of hardening time, the patient will be instructed on how to perform oral hygiene procedures and asked to refrain from eating or drinking for 30minutes.

In group I, the procedure repeated in $3^{\text {rd }}, 6^{\text {th }}$, $9^{\text {th }}$ and $12^{\text {th }}$ month as $\mathrm{T} 1, \mathrm{~T} 2, \mathrm{~T} 3, \mathrm{~T} 4$ respectively. In group II the procedure repeated in $6^{\text {th }}$ and $12^{\text {th }}$ month as T2 and T4 respectively. Digital vernier calliper used for measuring white spot lesions. MI varnish applied on $1 \mathrm{~mm}$ mesial, distal, occlusal and gingival to the surrounding brackets

\section{STATISTICAL ANALYSIS}

Comparison of the mean white spot lesions between the groups using mann-whiteny test is done. Comparison of the white spot lesions in group 1 within each quadrant at different time intervals using Friedman test and Comparison of the white spot lesions in group 2 within each quadrant at different time intervals using wilcoxon sign test and post-hoc wilcoxon sign test was done.

\section{RESULTS}

The 50 patients in each group I and II are examined in with interval of 3 months and 6 months respectively for one year. Reading are subjected to statistical analysis

Table-1: Comparison of the mean white spot lesions between the groups using mann-whiteny test $(\mathbf{t} 2)$

\begin{tabular}{|c|c|c|c|c|c|c|c|c|}
\hline T2 & Groups & $\mathbf{N}$ & Minimum & Maximum & Mean & S.D & Mean diff & p value \\
\hline \multirow{2}{*}{$1^{\text {st }}$ Quadrant } & Group 1 & 50 & 0 & 4 & .43 & .850 & \multirow[t]{2}{*}{0.43} & \multirow[t]{2}{*}{$0.001 *$} \\
\hline & Group 2 & 50 & 0 & 0 & .00 & .000 & & \\
\hline \multirow{2}{*}{$3^{\text {rd }}$ Quadrant } & Group 1 & 50 & 0 & 4 & 43 & 850 & \multirow{2}{*}{0.43} & \multirow{2}{*}{$0.001 *$} \\
\hline & Group 2 & 50 & 0 & 0 & .00 & .000 & & \\
\hline $2^{\text {nd }}$ Quadrant & Group 1 & 50 & 0 & 9 & 1.14 & 2.54 & 1.14 & $0.006^{*}$ \\
\hline
\end{tabular}


Irfan Habeeb et al; Saudi J Oral Dent Res, Jan 2022; 7(1): 12-17

\begin{tabular}{|c|c|c|c|c|c|c|c|c|}
\hline$\overline{T 2}$ & Groups & $\overline{\mathbf{N}}$ & Minimum & Maximum & Mean & S.D & Mean diff & p value \\
\hline & Group 2 & 50 & 0 & 0 & .00 & .000 & & \\
\hline \multirow{2}{*}{$4^{\text {th }}$ Quadrant } & Group 1 & 50 & 0 & 8 & 1.03 & 2.242 & \multirow{2}{*}{0.03} & \multirow{2}{*}{0.629} \\
\hline & Group 2 & 50 & 0 & 8 & 1.00 & 2.555 & & \\
\hline
\end{tabular}

Table 1 shows comparison of demineralization at 6 months. Number of measurement and mean value of degree of mineralization between group 1 , 2 in $1^{\text {st }}, 2^{\text {nd }}, 3^{\text {rd }} \& 4^{\text {th }}$ quadrant.
At 6 month, the incidence white spot lesions significantly varies among group I and group II in $1^{\text {st }}, 2^{\text {nd }}$ and $3^{\text {rd }}$ quadrants

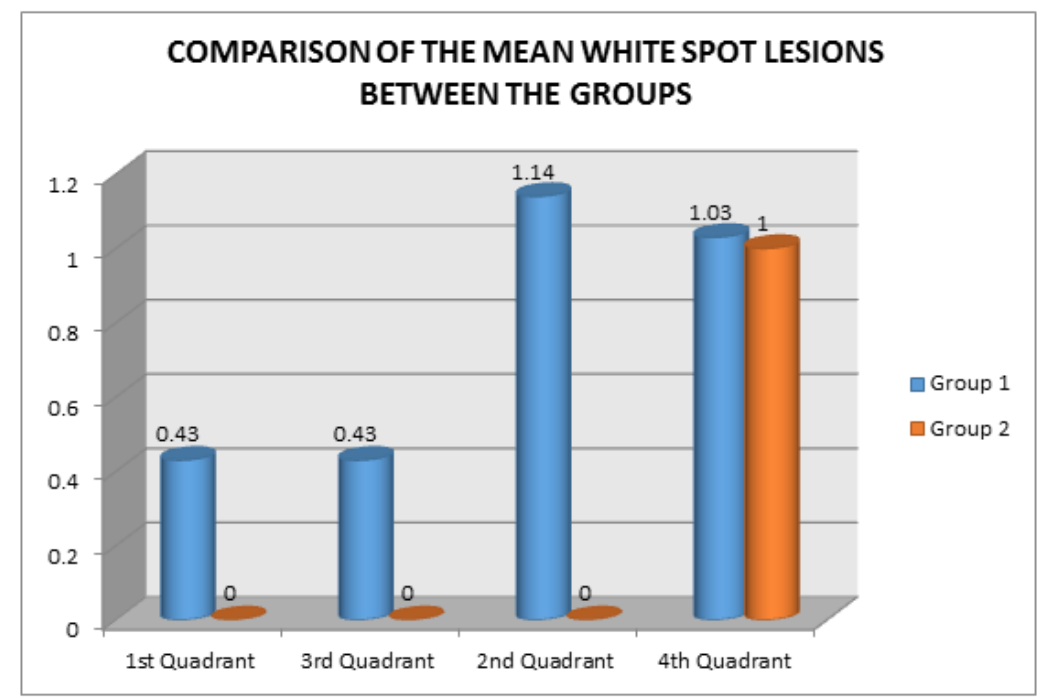

At 6 month, the incidence white spot lesions significantly varies among group I and group II in $1^{\text {st }}$, $2^{\text {nd }}$ and $3^{\text {rd }}$ quadrants

Table-2: Mean white spot lesions in group 1 (t3)

\begin{tabular}{|l|l|l|l|l|l|}
\hline & $\mathbf{N}$ & Minimum & Maximum & Mean & Std. Deviation \\
\hline $1^{\text {st }}$ Quadrant & 50 & 0 & 0 & .00 & .000 \\
\hline $3^{\text {rd }}$ Quadrant & 50 & 0 & 8 & .46 & 1.615 \\
\hline $2^{\text {nd }}$ Quadrant & 50 & 0 & 16 & 1.63 & 3.679 \\
\hline $4^{\text {th }}$ Quadrant & 50 & 0 & 14 & 1.20 & 3.225 \\
\hline
\end{tabular}

Table 2 shows comparison of white spot lesions in each quadrant in duration of 9 months among group1 patients. And there is no significance difference among each quadrant.

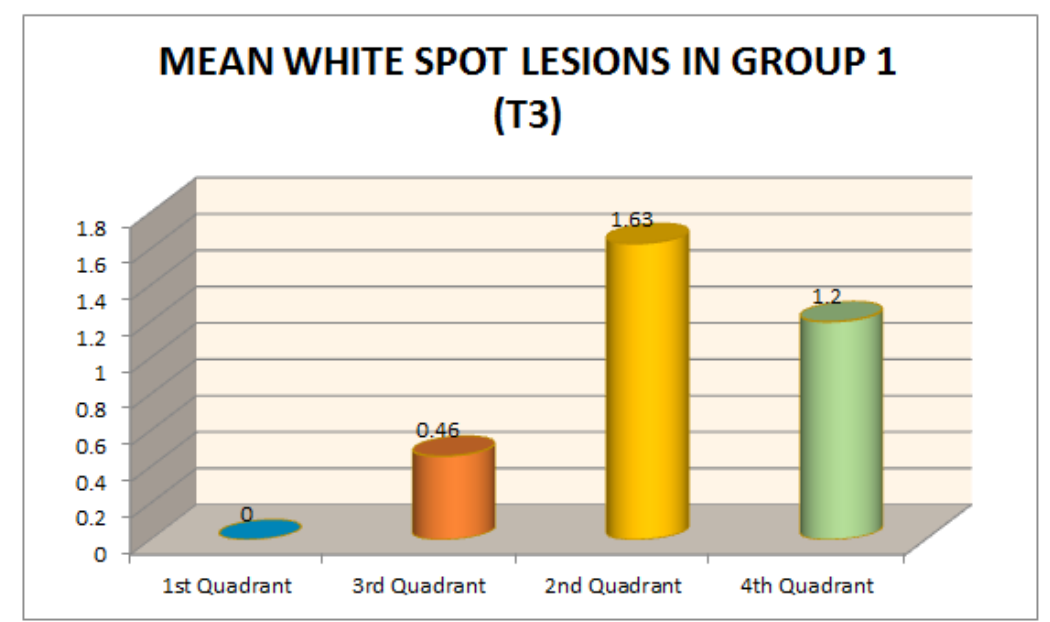


Table-3: Comparison of the mean white spot lesions between the groups using mann-whiteny test (t4)

\begin{tabular}{|c|c|c|c|c|c|c|c|c|}
\hline T4 & Groups & $\mathbf{N}$ & Minimum & Maximum & Mean & S.D & $\begin{array}{l}\text { Mean } \\
\text { diff }\end{array}$ & $\begin{array}{l}\mathbf{p} \\
\text { value }\end{array}$ \\
\hline \multirow[t]{2}{*}{$1^{\text {st }}$ Quadrant } & Group 1 & 50 & 0 & 0 & .00 & .000 & \multirow[t]{2}{*}{0.00} & \multirow[t]{2}{*}{1.00} \\
\hline & Group 2 & 50 & 0 & 0 & .00 & .000 & & \\
\hline \multirow{2}{*}{$3^{\text {rd }}$ Quadrant } & Group 1 & 50 & 0 & 10 & 1.06 & 2.543 & \multirow[t]{2}{*}{0.64} & \multirow{2}{*}{0.57} \\
\hline & Group 2 & 50 & 0 & 4 & .40 & 1.090 & & \\
\hline \multirow{2}{*}{$2^{\text {nd }}$ Quadrant } & Group 1 & 50 & 0 & 18 & 2.09 & 3.929 & \multirow[t]{2}{*}{1.25} & \multirow{2}{*}{0.08} \\
\hline & Group 2 & 50 & 0 & 7 & .74 & 1.837 & & \\
\hline \multirow[t]{2}{*}{$4^{\text {th }}$ Quadrant } & Group 1 & 50 & 0 & 10 & 1.29 & 2.585 & \multirow[t]{2}{*}{-0.34} & \multirow[t]{2}{*}{0.92} \\
\hline & Group 2 & 50 & 0 & 15 & 1.63 & 3.448 & & \\
\hline
\end{tabular}

Table 3 shows comparison of white spot lesions between groups $1 \&$ group 2 in each quadrant a t a duration of 1 year. And there is no significant difference among groups.

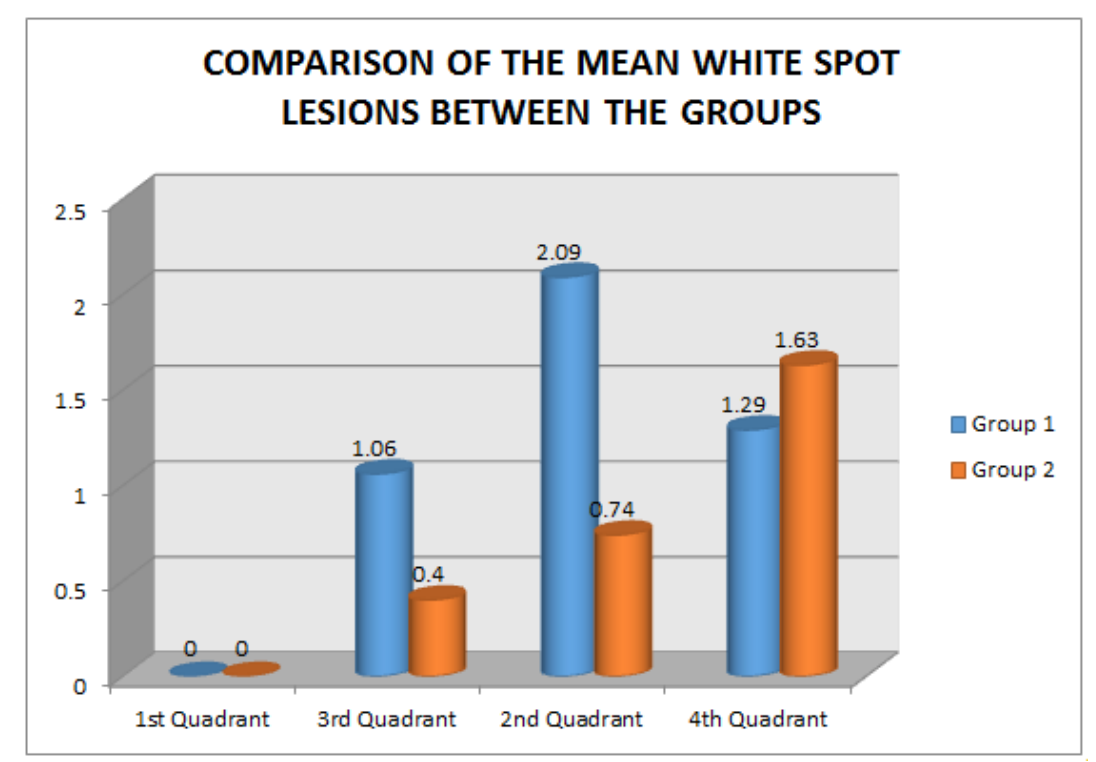

Table-4: Comparison of the white spot lesions in group 1 within each quadrant at different time intervals using Friedman test

\begin{tabular}{|c|c|c|c|c|c|c|c|}
\hline & & $\mathbf{N}$ & Minimum & Maximum & Mean & S.D & p value \\
\hline \multirow[t]{3}{*}{$1^{\text {st }}$ Quadrant } & $\mathrm{T} 2$ & 50 & 0 & 4 & .43 & .850 & \multirow[t]{3}{*}{$0.001 *$} \\
\hline & $\mathrm{T} 3$ & 50 & 0 & 0 & .00 & .000 & \\
\hline & $\mathrm{T} 4$ & 50 & 0 & 0 & .00 & .000 & \\
\hline \multirow[t]{3}{*}{$3^{\text {rd }}$ Quadrant } & $\mathrm{T} 2$ & 50 & 0 & 4 & .43 & .850 & \multirow[t]{3}{*}{0.097} \\
\hline & T3 & 50 & 0 & 8 & .46 & 1.615 & \\
\hline & $\mathrm{T} 4$ & 50 & 0 & 8 & .46 & 1.615 & \\
\hline \multirow[t]{3}{*}{$2^{\text {nd }}$ Quadrant } & $\mathrm{T} 2$ & 50 & 0 & 9 & 1.14 & 2.545 & \multirow[t]{3}{*}{$0.001 *$} \\
\hline & T3 & 50 & 0 & 16 & 1.63 & 3.679 & \\
\hline & $\mathrm{T} 4$ & 50 & 0 & 18 & 2.09 & 3.929 & \\
\hline \multirow[t]{3}{*}{$4^{\text {th }}$ Quadrant } & $\mathrm{T} 2$ & 50 & 0 & 8 & 1.03 & 2.242 & \multirow[t]{3}{*}{0.431} \\
\hline & T3 & 50 & 0 & 14 & 1.20 & 3.225 & \\
\hline & T4 & 50 & 0 & 10 & 1.29 & 2.585 & \\
\hline
\end{tabular}




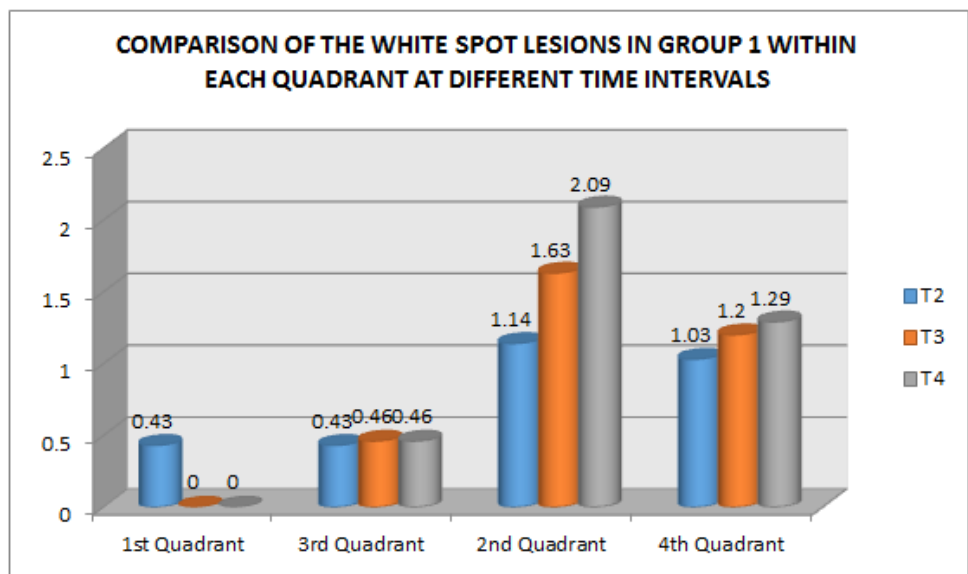

Table-4A: Post-hoc (wilcoxon sign test)

\begin{tabular}{|l|l|l|l|}
\hline & T2 V/S T3 & T2 V/S T4 & T3 V/S T4 \\
\hline $1^{\text {st }}$ Quadrant & $0.004^{*}$ & $0.004^{*}$ & 1.00 \\
\hline $3^{\text {rd }}$ Quadrant & 0.507 & 0.303 & 0.028 \\
\hline $2^{\text {nd }}$ Quadrant & 0.068 & $0.005^{*}$ & 0.059 \\
\hline $4^{\text {th }}$ Quadrant & 0.414 & 0.336 & 0.680 \\
\hline
\end{tabular}

$* p$ value significant at $0.05 / 3=0.016$

Table-5: Comparison of the white spot lesions in group 2 within each quadrant at different time intervals using wilcoxon sign test

\begin{tabular}{|c|c|c|c|c|c|c|c|c|}
\hline & & $\mathbf{N}$ & Minimum & Maximum & Mean & S.D & $\begin{array}{l}\text { Mean } \\
\text { diff }\end{array}$ & p value \\
\hline \multirow[t]{2}{*}{$1^{\text {st }}$ Quadrant } & $\mathrm{T} 2$ & 50 & 0 & 0 & .00 & .000 & \multirow[t]{2}{*}{0.00} & \multirow[t]{2}{*}{1.00} \\
\hline & $\mathrm{T} 4$ & 50 & 0 & 0 & .00 & .000 & & \\
\hline \multirow[t]{2}{*}{$3^{\text {rd }}$ Quadrant } & $\mathrm{T} 2$ & 50 & 0 & 0 & .00 & .000 & \multirow[t]{2}{*}{-0.40} & \multirow[t]{2}{*}{$0.042 *$} \\
\hline & $\mathrm{T} 4$ & 50 & 0 & 4 & .40 & 1.090 & & \\
\hline \multirow[t]{2}{*}{$2^{\text {nd }}$ Quadrant } & $\mathrm{T} 2$ & 50 & 0 & 0 & .00 & .000 & \multirow[t]{2}{*}{-0.74} & \multirow[t]{2}{*}{$0.027 *$} \\
\hline & $\mathrm{T} 4$ & 50 & 0 & 7 & .74 & 1.837 & & \\
\hline \multirow{2}{*}{$4^{\text {th }}$ Quadrant } & T2 & 50 & 0 & 8 & 100 & 2555 & \multirow{2}{*}{-0.63} & \multirow{2}{*}{$0.035^{*}$} \\
\hline & $\mathrm{T} 4$ & 50 & 0 & 15 & 1.63 & 3.448 & & \\
\hline
\end{tabular}

*significant

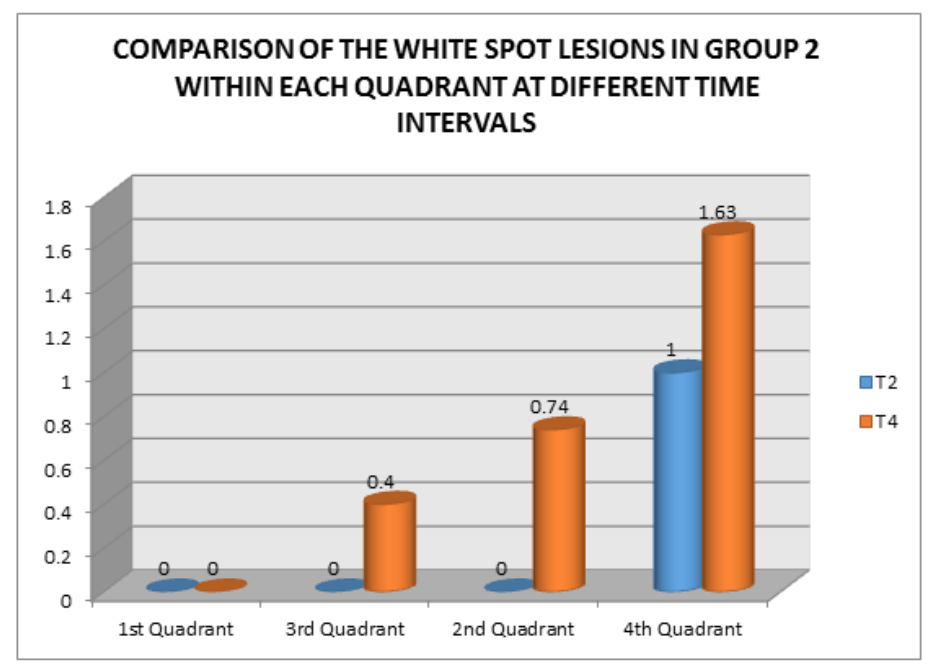


Table 1 shows comparison of demineralization at 6 months. Number of measurement and mean value of degree of mineralization between group 1 , 2 in $1^{\text {st }}, 2^{\text {nd }}, 3^{\text {rd }} \& 4$ th quadrant.

Table 2 shows omparison of white spot lesions in each quadrant in duration of 9 months among group1 patients.

Table 3 shows comparison of white spot lesions between groups $1 \&$ group 2 in each quadrant at a duration of 1 year. Table 4 shows comparison of white spot lesions in group1 within each quadrant to different time intervals. Table 4 A shows comparison of white spot lesions with respect to different intervals in each quadrant. Table 5 shows comparison of white spot lesions in group2 within each quadrant at different time intervals. Among group 1 and group 2 there is statistically significant difference when the MI varnish was applied 2 times or 4 times a year. At the 12 month time there is a significant differences in the demineralisation between treated and un treated teeth.

\section{DISCUSSIONS}

The white spot lesions surrounding the orthodontic brackets influence the aesthetic appearance of individuals. Once white spot lesions have appeared it should be treated accordingly. Remineralisation techniques involving gels, dentifrices, and mouth washes have been used. Combination of casein with fluoride in these products will increase the fluoride absorption and to certain extent it increases remineralisation. Hence, preventive procedures will be helpful rather than reparative lesion of white spot lesions. The choice of preventive procedures depends on patient safety, efficacy, ease of use and patient acceptance. MI varnish, fluoride varnish are the topical application which needs only little patient co-operation. Helfenstein U et al., conducted a meta-analysis study on comparing efficacy of fluoridated products with nonfluoridated products among 2700 children, have provided evidence of their great capacity to inhibit caries formation and showed higher caries prevention incidence in both permanent and deciduous dentition using fluoridated varnishes. Despite some variations in the percentages reported in these trails, they are between $38 \%$ and $46 \%$ [28].

\section{CONCLUSIONS}

MI varnish application significantly reduces white spot lesion during orthodontic treatment Periodic application of fluoride varnish tends to protect against white spot onset but not significantly so in patients with excellent oral hygiene. The protection offered by this MI varnish is statistically significant in both the maxillary and mandibular anterior teeth (central incisors), but not in the medial and posterior sectors. Additional protection is conferred by applying the product every 3 months compared with every 6 months.

MI varnishes, together with other prevention strategies, may be a useful aid to protect the enamel in noncompliant patients (those with a disability or poor hygiene education).

\section{REFERENCES}

1. Roopa, K. B., Pathak, S., Poornima, P., \& Neena, I. E. (2015). White spot lesions: A literature review. $J$ Pediatr Dent, 3(1), 1-7. 